\title{
Analysis of the Impact of Foldable Mobile Phones Design on People's Lives
}

\author{
Danny Ziyang Huang ${ }^{1}$ \\ ${ }^{1}$ Canada Qingdao Secondary School, Qingdao, Shandong, China 266000 \\ *Corresponding author. Email: dannyhzy@icloud.com
}

\begin{abstract}
Along with the development of smartphones, more and more different forms of cell phones are appearing, the sales of foldable screen cell phones are increasing, and the practicality of such phones has become a focus. In the face of this new technology, people have different opinions and opinions, so this paper will study the advantages and disadvantages of folding screen cell phones in daily use through screen manufacturing technology, screen material, software adaptation. The research topic of this paper is an analysis of the impact of foldable mobile phones on people's lives, which is studied by literature research method, functional analysis method, case study method, and observation method. The study concludes that along with the development of technology and the application of more and more new technologies, foldable mobile phones will eventually become a popular form of smartphones in our future.
\end{abstract}

Keywords: Technology, Smart Phone, Display Technology, Folding Screen, Mobile Phone.

\section{INTRODUCTION}

As the cell phone industry has evolved and various technologies have been updated, cell phones have become versatile. The annual Mobile World Congress in Barcelona and the Consumer Electronics Show in Las Vegas, known as the world's most effective mobile and consumer electronics shows, strongly influence the global communications industry. From the information released at the shows, the current "competitive focus" of the market is the launch of Huawei's mate x commercial folding screen phone based on fifth generation mobile communication technology (5g) and Samsung's Galaxy fold commercial folding screen phone. In addition, many technology vendors have also released stacked screen phone technology. [7] From the earliest flip phone and sliding, phone to touch screen phone in smartphones, and then to today's folding screen phone and rolling phone. With the folding-screen phone, a new type of phone, coming into our lives, people cannot help but have various questions, such as whether the shape of this phone is solid, whether it can meet regular use, or whether it is easy to use. Therefore,the customer hopes to find out all the problems encountered in the daily use of the folding screen mobile phone through research, and then find the type of the problem. By understanding the type of the problem, people can also solve the current technical defects. This article will explain the impact of folding mobile phones on human society by introducing the development and characteristics of folding mobile phones.

\section{OVERVIEW OF FOLDABLE PHONES}

With the development of the mobile phone industry and the update of various technologies, the form of the mobile phone has become changeable. From the earliest flip phones and slide phones to touch screen phones in smartphones to today's folding screen phones and scroll phones. As the new type of mobile phone with folding screen enters human life, people have many uncertainties about the performance of the mobile phone, such as whether the shape of this mobile phone is sturdy, whether it can meet daily use, and whether it is convenient to use. This article wants to discuss whether the folding screen mobile phone can satisfy our daily mobile phone use.

\subsection{Definition of foldable mobile phone}

The academic article said that: "A foldable phone is a smartphone with a unique display that can fold in half, much like a sheet of paper. In 2011, Samsung first started talking about these bendable displays that could be folded or rolled, the first foldable phone was unveiled on 2018. Flexible screens are not new. The author has been watching them collectively for years, dreaming of the day people could have devices that benefit from that 
flexibility. Nevertheless, it has taken years to see even a tiny bit of the capabilities allowed by that technology." [1] In short, a folding screen mobile phone is a kind of mobile phone that uses a soft screen and then can change its shape by folding the screen. People can use a smaller size through this technology, making the large screen mobile phone more portable. People could know the "Foldable Phone" idea is entirely perfect that could have big small in a small phone. But there is still an endless stream of people who want to buy. The data shows that: Manufacturers shipped a total of 1 million foldable smartphones in 2019, and forecasts show that their shipments will reach 8 million in 2020. The shipments of collapsible smart phones in 2019 accounted for only a small part of the global shipments of 1.37 billion smart phones that year. Entering the market in 2019, analysts predict that the global shipment of foldable smartphones will reach 50.1 million by 2022. [2] This shows that many people still choose to buy folding screen mobile phones, so people need to investigate the rationality of folding screen mobile phones in daily life from many aspects.

Now many mobile phone manufacturers have launched this folding screen mobile phone. The folding methods of general folding screen mobile phones are roughly divided into entropion and eversion. The first one that I would like to introduce is the Samsung Galaxy $\mathrm{Z}$ fold, which is the newest generation of Samsung phones that uses a fold in screen. Through the support of nano glass and foot chains, it can meet the daily use of a mobile phone. This kind of design is widely used, such as Huawei's mate $\mathrm{x} 2$ mobile phones and Mi fold mobile phones. Nevertheless, the coach design and screen design of different manufacturers are not the same. This kind of folding design can ideally ensure the safety of the inner screen, reduce the damage to the screen itself caused by folding times, and reduce the apparent degree of creases. Although this design sounds excellent, there are still some shortcomings. The article said that: The Galaxy $\mathrm{Z}$ Fold 2 is not just a foldable phone. This is a movement waiting to happen. Samsung's successful remake of its first foldable design has improved the original Galaxy fold in almost all aspects. It starts with the new 6.2-inch outer screen, continues to the 7.6-inch inner screen and each hinge part, groove and gap in the middle, provides a high-end device, and leads the full-size foldable mobile phone from the fantasy field to the edge of reality for the first time [3]. Because of the display of materials, this kind of folding will cause much damage to the mobile phone. Compared with the early generation of mobile phones, the firmness of mobile phone screens has been dramatically improved, but there are still some problems.

\subsection{Categories of foldable phones}

The following way to fold a mobile phone is to fold it outwards; that is, the mobile phone has a large screen and then fold it back to the back of the mobile phone to complete the folding. This design is represented by Huawei's first-generation folding screen mobile phone. On February 24, Barcelona, Spain, Huawei unveiled its first $5 \mathrm{G}$ folding screen phone, the Huawei Mate $\mathrm{X}$, with an 8 -inch body size that folds out asymmetrically. Huawei Mate $\mathrm{X}$ is equipped with $5 \mathrm{G}$ signal support and equipped with Baron 5000 + Kirin 980 cores, allowing users to have ultra-high speed. Huawei Mate $\mathrm{X}$ is equipped with $5 \mathrm{G}$ signal support with Baron $5000+$ Kirin 980 chip, allowing users to have an ultra-high speed experience with a peak of $4.6 \mathrm{Gbps} / \mathrm{sec}, 1 \mathrm{G}$. Huawei X can download a movie in 3 seconds. Huawei claims that it is the world's fastest $5 \mathrm{G}$ folding phone and the thinnest and lightest folding phone. [5] The advantage of this design is that it can reduce the production cost of the mobile phone because it has only one external screen and no second screen, which can become very thin when the mobile phone is opened. This is very convenient to use because folding phones are usually cumbersome. Nevertheless, this kind of screen also has many shortcomings. We know that the screen of a regular smartphone is very afraid of falling, and the friction between the screen and other problems in daily use will also cause many scratches on the screen. Then, the mobile phone turned backwards will make the other side of the screen become the back of the mobile phone. Ordinary intelligent phones cannot resist the damage caused by long-term use, not to mention the soft folding screen of mobile phones.

Definitely, the third common folding form is the Samsung Z flip. It is very similar to the first folding form, which is also inverted, but it has no screen on the surface of the mobile phone to operate the mobile phone, only a small screen to display the mobile phone notice. He is Turning Ordinary smartphones inward. Reduce the size of the phone. This kind of mobile phone has a considerable advantage, that is, the folding area is small. Because the folding area is small, the crease caused by the folding area is relatively less noticeable, and the probability of damage is relatively small. Nevertheless, in the face of this design, people have to open the mobile phone screen before they can operate and read the mobile phone's content. Nevertheless, the price of this design is relatively low, and it can also have a lower maintenance cost when the product is artificially damaged. Therefore, this way has become the most crucial channel for ordinary people to start folding screen mobile phones.Science drives the progress of theory, and the progress of theory promotes the development of engineering. Engineering lays the foundation for design - the emergence of design changes and benefits human life. A good design can make products bring a different experience to people. 


\section{INFLUENCING FACTORS OF USING FEELING OF FOLDABLE MOBILE PHONE}

In the design of folding screen mobile phones, four key factors affect mobile phone use in our life. Screen manufacturing technology, screen materials, foot chain design technology and software optimisation will make us get a different experience using mobile phones.

\subsection{Screen manufacturing technology}

The screen manufacturing technology of mobile phones is essential. Good screen manufacturing technology can ensure that the mobile phone screen can meet the regular use of our mobile phone. The screen can be folded because it can be folded when an object is fragile. Most of today's folding screen phones use fragile glass so that the screen can be folded. So advanced manufacturing technology can make the screen and glass better fit, reduce the probability of damage. For example, Samsung's Z flip phone uses unique product technology to ensure the screen's usability. Because people know that when the screen is fragile, it means that the screen is straightforward to be damaged, maybe when people touch the screen hard, the screen will be broken. So what does Samsung do? It is covered with plastic on $0.7 \mathrm{~mm}$ thick glass. This layer of speed can ensure that the screen can be well protected without direct contact with the outside world.[4] Of course, this technology also has some defects, such as the thickness of the mobile phone becomes thicker, it is easy to leave irreversible scratches on the screen of the mobile phone. However, with the development of new manufacturing technology, the hardness of glass will continue to increase, so it does not need to use plastic for protection. At that time, the problem of folding screen mobile phone screen will also be solved. People can use the folding screen mobile phone as a standard mobile phone and will not use it carefully because the screen is easy to damage.

\subsection{Screen Material}

The use of screen materials has also become a focus when people see the new folding screen mobile phone. The use of more advanced material technology can make the mobile phone price cheaper and make the mobile phone's service life higher, not because of the accidental damage of the screen and lead to the scrapping of the mobile phone. The article said that: The main problem is this: People can only stretch a material so far in its chemical bonds before it breaks, a concept known as tensile strength. However, if people use a thinner sheet of glass, people stretch less material in the same space, bend fewer layers and stretch fewer chemical bonds, Mydlak says. That puts less tensile stress on the glass and lets people bend it tighter before it breaks. Making the glass thinner is one of the "two main tricks" for making it bend, because physics students learn that tensile strain is linearly related to thickness, said Juejun $\mathrm{Hu}$, an associate professor of materials science at MIT. When you reduce the thickness of glass to less than 100 microns -- about the thickness of a human hair -- that's when it can be bent large enough to be used for necessary folding gadgets, experts say. In order to prevent the cell phone display by external oxygen and water vapor oxidation and corrosion, in the display of the most surface also covered with a protective layer, this technology is called encapsulation. Traditional cell phones are encapsulated with glass in the top layer, but the rigid glass package is also not suitable for flexible folding screen. One of the main solutions is called thin film encapsulation (ThinFilmEncapsulation, or TFE). As the name implies, this is a thin, light-transmitting and corrosion-resistant plastic film as a protective film for cell phone screens. [11] If people want a phone that folds inward without a huge hinge gap, people are talking tens of microns. When people have perfect materials, people can get a thin and solid mobile phone screen.

\subsection{Foot chain design technology}

The anklet design of the mobile phone is also fundamental. The function of the foot chain is to protect the mobile phone screen, which can be stored entirely in the folding place of the mobile phone. The foot chain connects the two parts of the phone and also supports the screen. In the earliest research, the mobile phone screen was responsible for linking the two parts of the mobile phone. Nevertheless, with the development of the foot chain technology, people find that only using the screen to connect the mobile phone will make the mobile phone's appearance very ugly and cause the screen to break easily and the mobile phone to be damaged. After the folding screen, mobile phone joined the anklet, the integrity of the mobile phone has been dramatically improved. First: it is quite hard. The rigidity of this hinge increases the overall sense of trust and durability, but it also makes it difficult to turn out the phone with one hand. I can do this, but I need more strength than I feel comfortable, as if it might fly out of my hands. Most of the time, it's more comfortable to open $\mathrm{Z}$ flip with two hands. This is twofold, because if people wedge people thumb to start the flip action, there is a risk that people may damage the screen plastic under the thumb nail.[4] From then on, people know that although the existence of the foot chain will increase the reliability of the folding screen mobile phone, the relatively high price and greater weight may make the Department people not buy the mobile phone. Nevertheless, with the development of mechanical engineering, the lightweight design of the foot chain will make the design of mobile phones more excellent. 


\subsection{Software Optimisation}

The last factor affecting the use of folding screen mobile phones is software optimisation. people know that the screen ratio of most mobile phones is about 21.5:9, but when the folding screen mobile phone is unfolded, the screen ratio will be similar to $1: 1$. So the optimisation of the software is crucial. A sound system can improve the experience of the whole folding screen mobile phone. The current solution is divided into two parts. First of all, the system, because after turning on the mobile phone, the screen becomes more extensive, time, power, control centre, screen home page, etc., will increase with the larger screen. Now Android system and Huawei's Hongmeng system have an excellent design. Mi has its solution. It designed a unique system; when the screen is produced, it can become a system similar to the computer operation interface, with more convenient operation logic and better interaction design. The second part is the application. Because with the enlargement of the screen, the application should have its adaptation. A good adaptation can make full use of the ample space to display more content instead of blindly enlarging the screen. Now WeChat and other applications have completed optimising most folding screen mobile phones, making the experience higher.

\section{DISCUSSION}

Because of the difference between the design and the manufacturer, various manufacturers 'unique designs on the market also optimise the experience of folding screen mobile phones. In 2012, China became the single largest market in the world in the cell phone sector. [8] At present, in addition to Huawei, the manufacturers that have officially launched folding screen phones in the domestic market include Samsung, Motorola and Wakyu Technology. They cut into the market from different angles and explore the possibilities of development. [10] Because of the huge mobile phone market in China, major manufacturers are striving to use special designs to attract customers. First of all, Huawei has a robust design and has unique views on mobile phones. When the first generation folding screen mobile phone was released, there was a button to expand the screen on the back of the mobile phone. Although people always think that folding-screen phones should be easier to open, it is not. That button fastens the screen on the backside of the phone to prevent damage caused by accidental deployment. The latest mobile phones have a better design. Because after the mobile phone is folded, people will find that the thickness of the foot chain is significant, and it will make people feel particularly uncomfortable when the mobile phone is put in the pocket.

Nevertheless, if the thickness of both ends of the mobile phone is the same, then it is an excellent design. Huawei's third-generation folding screen mobile phone changes the thickness of the mobile phone, like a trapezoid, so that all parts of the mobile phone have the same thickness after folding. Of course, Samsung mobile phone also has its unique solution. Because the Z flip phone has only one screen, it is not easy to see the phone's information when the phone is folded. Samsung mobile phone designs a small screen on the back of the mobile phone, displaying time, messages, phone notifications, music playing, etc. In the case of costsaving, it provides us with a more convenient and excellent mobile phone experience. These designs will also make the use of mobile phones more and more humanised.

Hence, the advantages of folding screen phones make customers willing to buy. First, the design of the big screen. This is the most intuitive advantage that folding screen mobile phones can bring us; it has a very portable way to give us a giant screen and a perfect user experience. When people watch and chat, the bigger screen will bring us a perfect visual experience. Of course, for offices, the excellent split-screen function can also make office and learning more convenient. The second aspect is the symbol of identity. People will judge a person's life with charm, high identity, or a passion for technology based on what a person owns. First of all, the price of a folding screen mobile phone is relatively high, and the use of a folding screen mobile phone means that it has a unique identity and social status. Folding screen mobile phone because of its excellent performance, more convenient considerable screen experience, can also reflect a person's love for life. A person is always willing to use fresh food to enrich his life, which embodies a person's interest in life. Of course, this is also a new technology product, and it is also the first choice for people who love technology to use their mobile phones. A variety of innovations make him occupy a more important position in our life.

Mobile phones are not perfect devices. Experts said that the second thing is that Samsung explained the rigidity of the hinge by pointing out that you can put the phone on a table half open, like a very small laptop. For some reason, it is called "flex mode," and the idea is that people can use it to make video calls or take pictures. Right now, only a few apps support it. I mostly think it's a gimmick: propping people's phone up and having the camera point where people want it is not a problem that requires a $\$ 1380$ solution. [4] Folding screen products in the market after two years of testing, the overall experience have been a big upgrade and progress. The biggest problem right now is the high cost, imagine, if the price of folding screen phones to the current level of flagship phones, that buy the people estimated Will be more, and I believe this day is not far away. [9] This is the first drawback of mobile phones: the price is too high. Although this mobile phone has a variety of excellent performances, these performances in the face of their price point of view look pretty useless. Nevertheless, 
with technology, screen technology and chain technology, the price will undoubtedly decline. The second disadvantage is that mobile phones are straightforward to damage. Because of the unique screen mobile phone, the mobile phone in the face of a slight bump will also appear colossal damage. Not only is it hurt by the outside world, but the mobile phone's screen will also have apparent creases after folding for a very long time. Third, the maintenance cost of this mobile phone is very high. In the face of parts damage, the mobile phone parts have no maintenance value; intelligent replacement significantly increased the cost of use. Forth, this kind of mobile phone is also cumbersome and has a short standby time. Folding screen phone can bring us excellent visual viewing art effect, but the body thickness and standby time is currently insurmountable problems[6].

Although the mobile phone has a variety of shortcomings, with the development of technology, more and more advanced manufacturing technology, more and more mature material science, and excellent mechanical engineering design, coupled with the improvement of software interaction and overall innovative design, folding screen mobile phone will occupy a crucial position in our life.

Engineering and design should not be separated, they should influence each other The development of science and technology drives the progress of engineering, which lays the foundation for design. Design engineering is to apply engineering to people's lives in a unique way. The appearance of the folding screen mobile phone is a combination of the development of material science, chemistry and other technologies, which drives the development of screen material technology. Since then, the screen is no longer fixed but can be folded. Facing the application of mechanical engineering, electronic and electrical engineering, special mechanical foot chain design and unique circuit design are born. With the birth of these technologies, the design integrates them, with the help of the food chain and the screen with unique materials, plus the user experience and aesthetic perception of the appearance of products in interaction and industrial design, which creates the birth of folding screen mobile phone, which is a very typical embodiment of the development of engineering and design driving the progress of human life.

\section{CONCLUSION}

Generally speaking, a folding screen phone has many drawbacks. However, from it, people can see the development of technical engineering and the progress of the design. The combination of people and technology is the highest level people pursue. Technology changes people's lives. This is what everyone expects. The folding screen phone carrier has an excellent engineering design and an excellent interactive experience in our lives. people hope that the efforts of engineers can be translated into the optimisation of our life experience. The folding screen phone is a typical example. As technology science advances and more and more excellent designs emerge, folding-screen phones will change the entire cell phone market and become the most widely used form of cell phones. Folding screen cell phones will eventually become a success. This article is not comprehensive because of the limitation of the research method, and it only analyses this issue from the theoretical basis, article, and technology. This article can be improved by adding questionnaires, interviews, etc., to collect feedback and demand from all cell phone users and potential customers, through which people can better find the advantages, disadvantages and problems of cell phones. After that, the research point should be focused on optimisation above. Cell phone form has been determined, and then the next time should seek the current form of the problem, and then through these problems, complete a better design and optimisation so that it can be more adapted to people's lives. There are many research directions in the future. First, the screen technology should develop a better coating, better panel technology, and reduce the damage of daily use. The second aspect is to improve the design of the hinge or use other ways to fix the two parts of the phone to increase its durability. The third aspect is the adaptation and optimisation of applications so that the use of cell phones have a better experience.

\section{ACKNOWLEDGMENT}

I would like to show my respect to the people who helps me a lot about writing this article. I would like to thank to my professor who gave me the aim and idea about this article. I also want to show my respect to the teacher who helps me find the structural problem. Without them, I won't finish it easy like this.

\section{REFERENCES}

[1]. Ledford, Jerri. "Foldable Phones: This Is Not Your Father's Flip Phone." Lifewire, 22 Nov. 2019, www.lifewire.com/what-is-a-foldable-phone4178374.

[2]. Published by S. O'Dea, and Jun 15. "Global Foldable Smartphone Shipments in 2019, 2020 and 2025." Statista, $15 \quad$ June 2021, www.statista.com/statistics/1101618/globalfoldable-smartphone-shipments/.

[3]. Dolcourt, Jessica. "Galaxy Z Fold 2: All the Ways This Foldable Do-over Improves on the Original." CNET, www.cnet.com/tech/mobile/samsunggalaxy-z-fold-2-all-the-ways-this foldable-do-overimproves-on-the-original/. 
[4]. Bohn, Dieter. "Samsung Galaxy Z Flip Review: Temper Your Expectations." The Verge, The Verge, 21 Feb. 2020, www.theverge.com/2020/2/21/21146465/samsunggalaxy-z-flip-review-folding-glass-flip-phonescreen-camera-price.

[5]. Xuwei, Lin. (2019) "Huawei releases the first 5g folding screen mobile phone." East China Science \& Technology, 3(1), 9 .

[6]. Yumeng Li. (2019) "Folding screen mobile phone is eager to break through the problem -- paper battery." China Non-Governmental, 4(1), 76.

[7]. Dahong Deng. (2019) "This year is the "first year of the outbreak" of folding screen mobile phones." Business China, 5(1), 14-16.

[8]. Feng, Li . (2021) "The underlying logic of new consumption behind the rise of Huawei, Dyson and three and a half tons" CBMAG, 7(1), 25-27 Popular Computer Week, 7(13), 1-2.

[9]. Observation . (2021) "Under screen technology broke out and the folding screen market grew" Popular Computer Week, 7(13), 1-2

[10].Fan, Zhang . (2021) "Folding screen - the new blue ocean of cell phone market" China Brand, 4(1), 7071 .

[11].Jiaweilong, Tie . (2021) "How difficult it is to make the phone screen "curved"" Technology Fans, 3(1), 22-23. 